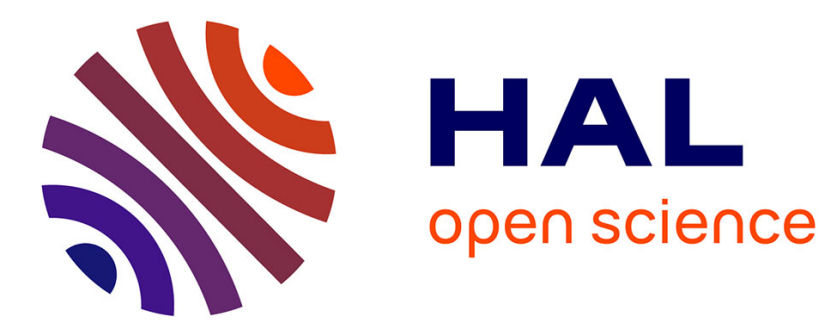

\title{
Torque in Taylor-Couette flow of viscoelastic polymer solutions
}

\author{
Borja Martínez-Arias, Jorge Peixinho
}

\section{To cite this version:}

Borja Martínez-Arias, Jorge Peixinho. Torque in Taylor-Couette flow of viscoelastic polymer solutions. Journal of Non-Newtonian Fluid Mechanics, 2017, 247, pp.221 - 228. 10.1016/j.jnnfm.2017.07.005 . hal-01919472

HAL Id: hal-01919472

https://hal-normandie-univ.archives-ouvertes.fr/hal-01919472

Submitted on 29 May 2019

HAL is a multi-disciplinary open access archive for the deposit and dissemination of scientific research documents, whether they are published or not. The documents may come from teaching and research institutions in France or abroad, or from public or private research centers.
L'archive ouverte pluridisciplinaire HAL, est destinée au dépôt et à la diffusion de documents scientifiques de niveau recherche, publiés ou non, émanant des établissements d'enseignement et de recherche français ou étrangers, des laboratoires publics ou privés. 


\title{
Torque in Taylor-Couette flow of viscoelastic polymer solutions
}

\author{
Borja Martínez-Arias, Jorge Peixinho \\ LOMC, CNRS and Université Le Havre Normandie, 76058 Le Havre, France
}

\begin{abstract}
When a polymer solution is sheared between concentric cylinders, with the inner rotating and the outer fixed, the torque on the inner cylinder is modified compared to a Newtonian fluid of the same viscosity revealing the different flow patterns that emerge above the linear stability threshold for circular Couette flow. Here, mixtures of relatively short and long linear polymers in dilute and semi-dilute concentrations were considered. Their shear viscosity and extensional relaxation time are quantified. The stability of the flow is monitored through torque measurements and flow visualisations for a constant rate of acceleration and deceleration of the inner cylinder in a wide range of polymer concentrations. The torque exhibits an hysteretic behaviour, typical of subcritical transition. For large concentrations, six different numbers of steady solitary pairs of vortices, called diwhirls, were observed depending on the deceleration rate and their torque contribution is reported.
\end{abstract}

Keywords: Torque, Taylor-Couette flow, Rheology of polymer solutions

\section{Introduction}

The stability of complex fluids in flows with curved streamlines is often considered in order to enhance mixing, for instance in serpentine microflows [7] or flow reactors [39]. Hence, the Taylor-Couette flow, i.e., the flow between concentric differentially rotating cylinders, can be destabilised by centrifugal forces when the rotation rate of the inner cylinder exceeds a critical value. When filled with a viscoelastic polymer solution, rheological properties can give rise to the inertio-elastic instabilities, or elastic instabilities [26], and special patterns, such as localised steady vortex pairs of large wavelength, 
called tall Taylor cells $[5,20]$ or diwhirls $[17,18]$ can arise. The challenge when dealing with polymer solutions is the increase of dimensionality of the parameter space, including parameters depending on the rheological properties of the fluid.

After pioneering studies in the sixties [11, 26], there has been a growing interest in viscoelastic instabilities in the Taylor-Couette system $[26,17$, 19, 9]. Theoretical studies are nowadays complemented by numerical calculations using viscoelastic models, like the upper-convected-Maxwell fluid model [36] or the Oldroyd-B model [25] and also finite extensible nonlinear elastic (FENE) dumbbell models [24, 47, 29, 30]. Although these studies were able to reproduce many flow properties observed in experiments, such as diwhirls, the quantitative comparison in torque and wavelength selection is not well understood.

From an experimental point of view, there is a large variety of polymers and the present study considers high-molecular-weight linear polymer poly(ethylene oxyde) (PEO). The properties of aqueous PEO solutions and mixtures of PEO and poly(ethylene glycol) (PEG) are well documented $[3,9,23,8,48,41,6,2,22,14]$ and stability diagrams of different regimes in Taylor-Couette flow are available $[9,14,27]$. Recently, Dutcher and Muller $[13,14]$ summarised most of previous experimental studies on viscoelastic Taylor-Couette flow. They identified inertial and inertio-elastic transitions depending on the viscoelasticity of the fluids (viscosity ratio and Elasticity number, defined below). Another technique to detect the transition is to monitor the torque that the fluid exerts on the inner cylinder as done by Denn and Roisman [11], Yi and Kim [50] and Groisman and Steinberg [19]. Denn and Roisman [11] provided torque data for several concentrated polymer solutions, including PEO of $4 \times 10^{6} \mathrm{~g} / \mathrm{mol}$. Yi and Kim [50] studied dilute polyacrylamide solutions. Groisman and Steinberg [19] also studied polyacrylamide in sugar syrup solutions and reported hysteretic behaviour for a single fluid. The novelty of the present study lies on systematic measurements of the torque in a wide range of polymer concentration. The advantage of the monitoring of the flow through torque is the possibility of quantifying the hysteresis in acceleration and deceleration protocols hence quantify the subcritical behaviour.

Concentrated polyacrylamide viscoelastic solutions are known to exhibit solitary vortex pairs solutions known as diwhirls [5, 20, 17] for Taylor-Couette flow of radius ratio 0.8 . The most detailed studies of these solutions are by Groisman and Steinberg $[17,19]$ where the radial velocity was measured and 
they showed that diwhirls are really a pair of vortices having a common core of fast fluid motion inwards. The inward radial component in the core flow is relatively fast compared to the slow outflow, which spreads axially over many gap widths. They also showed diwhirls can merge and different number of diwhirls can be observed. These diwhirls solutions were reproduced in threedimensional time-dependant numerical simulations of viscoelastic FENE-P models [47], where the drag was found to be two to three times the laminar drag. Note that similar fluid systems (POE solutions) have been shown to exhibit drag reduction, but for low concentrations and far from the transition at relatively large velocities $[38,1]$.

Our objective was to carry out a study of the dependence of the flow patterns and the torque on different rotation speeds during slow acceleration/deceleration cycles. A brief description of the experimental setup and the working fluids is given in section 2 . This is followed, in section 3 , of rheological results on the fluid. In section 4 , the results that consist of flow diagrams and the associated torque measurements are presented and discussed. The wavelength and the torque of the diwhirls are found to be controlled by the deceleration rate of the inner cylinder.

\section{Experimental methodology}

\subsection{Apparatus}

A controlled stress rotational rheometer (Anton Paar MCR 501) was used with different geometries. For measuring the dynamic shear viscosity, $\mu$, a bob-cup geometry was installed having the bob radius $13.330 \pm 0.001 \mathrm{~mm}$ together with a cup of radius $14.460 \pm 0.001 \mathrm{~mm}$ and a length of $40.006 \pm 0.001$ $\mathrm{mm}$. The Taylor-Couette geometry is also fitted on this rheometer and a sketch of the experiment is presented in figure 1 . The radii of the inner and outer cylinders are $r_{i}=50.00 \pm 0.01 \mathrm{~mm}$ and $r_{o}=55.00 \pm 0.01 \mathrm{~mm}$, respectively. The gap between the cylinders is $d=r_{o}-r_{i}=5.00 \pm 0.01$ $\mathrm{mm}$. The length of the inner cylinder is $L=150.0 \pm 0.5 \mathrm{~mm}$. Thereby, the geometrical dimensionless parameters of the Taylor-Couette system are the radius ratio $\eta=r_{i} / r_{o}=0.909$ and the aspect ratio $\Gamma=L / d=30$. The ends of the gap and the outer cylinder are stationary walls, only the inner cylinder is moving. The rheometer allows to measure the angular speed of the inner

cylinder, $\Omega$, and the torque, $T$, that it exerts on the fluid. The accuracy of the torque is $0.5 \%$ of the measured value and is never smaller than $0.2 \mu \mathrm{N}$.m. 


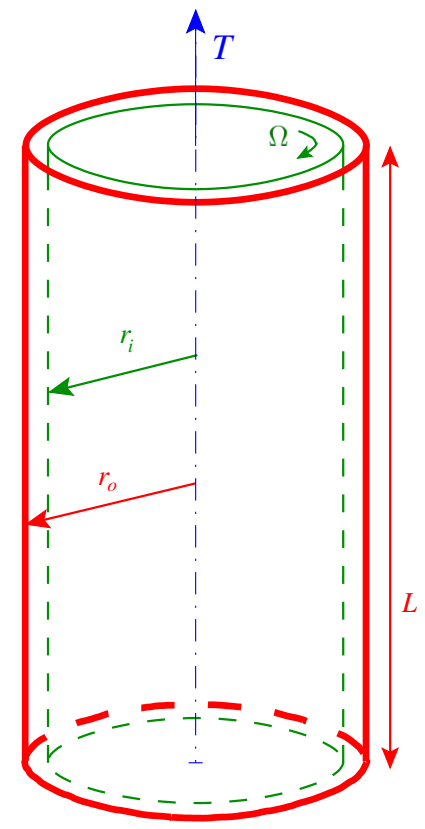

Figure 1: Schematic diagram of the Taylor-Couette experiment (drawn to scale).

There is a glass jacket around the outer cylinder connected to a flow of water in order to maintain the temperature at $22 \pm 0.05^{\circ} \mathrm{C}$.

The outer cylinder and the jacket are made of glass, thus flow visualisation can be used. Snapshots (see figure 8) are obtained using a six megapixel SLR digital camera together with illumination from the top. For the spatio-temporal diagrams (see figures 5 and 6), flow visualisation images are recorded at $15 \mathrm{~Hz}$ using a digital digital camera with over 1000 pixels in the vertical direction. Then, a computer program combines vertical-pixel lines from the mid-gap radius at different times. Further details of the device and the measurement systems can be found in $[32,33]$.

The inertia effects are measured by the Reynolds number, $R e=\dot{\gamma} \tau$, where $\dot{\gamma}=\Omega r_{i} / d$ is the applied shear rate and $\tau=d^{2} / \nu$ is the viscous diffusion time across the gap, where $\nu$ is the kinematic viscosity of the working fluid. In addition, the Taylor number, $T a$, is sometimes used as a measure of the centrifugal forces and the conversion of $R e$ is the following, $T a=$ $\operatorname{Re} \sqrt{(1-\eta) / \eta}[9]$. The torque exerted on the inner cylinder can be made dimensionless as $G=T /\left(2 \pi \rho \nu^{2} L\right)$ where $\rho$ is the density of the working fluid. 
The ratio

$$
N_{\omega}=\frac{G}{G_{l a m}}, \quad \text { where } \quad G_{l a m}=\frac{2 \eta}{(1+\eta)(1-\eta)^{2}} R e
$$

represents the transfer of the momentum in the radial direction and is sometimes called pseudo-Nusselt number in analogy with the thermal convection. $G_{l a m}$ is the torque of the laminar flow for an infinitely long cylinder [49]. For Newtonian solutions, it is related to the dissipation rate [45]. The product of the applied shear rate, $\dot{\gamma}$, and $\lambda_{e}$, the extensional relaxation time, is the Weissenberg number, $W i$. Finally, the elasticity number is $E l=\lambda_{e} / \tau$.

\subsection{Working fluids and experimental protocol}

The working fluids are mixtures of water, PEG, PEO and isopropyl alcohol (IPA). They are transparent, non-ionic and non-toxic. The PEG is a polymer with relatively short linear chains whose molar mass is of $20000 \mathrm{~g} / \mathrm{mol}$. The PEO polymer, also from Sigma Aldrich, has a molar mass $M_{w}=8 \times 10^{6} \mathrm{~g} / \mathrm{mol}$. These polymers have the simplest structure: $\mathrm{H}-\left[\mathrm{O}-\mathrm{CH}_{2}-\mathrm{CH}_{2}\right]-\left[\mathrm{O}-\mathrm{CH}_{2}-\mathrm{CH}_{2}\right]-[\ldots]-\mathrm{OH}$ among water-soluble polymers, which allows a wide range of concentrations and a large number of macromolecular conformations.

Considering the mixture of water with $7 \mathrm{wt} . \%$ PEG as the solvent, it is possible to estimate an overlap concentration, $c^{*}$, of the POE polymer using the relationship proposed by Graessley $[15]: c^{*}=0.77 /[\eta]$, where $[\eta]$ is the intrinsic viscosity. Bailey [3] reported previously measured intrinsic viscosity for POE: $[\eta]=0.072 M_{w}^{0.65}$. Although this correlation was obtained for slightly different conditions, it leads to an estimate of $c^{*} \simeq 350 \mathrm{ppm}$, which is consistent with previous results $[42,23,2]$. Five PEO concentrations have been chosen that range from dilute and semi-dilute regimes. The density of the mixture is $1009 \pm 1 \mathrm{~kg} / \mathrm{m}^{3}$ and, in due course, 2 wt.\% of Kalliroscope was added for visualization purposes. All fluids were prepared in batches of $1 \mathrm{~kg}$ and the protocol is as follows. In one vessel, PEG flakes were dropped in water and the mixture was stirred gently until complete dissolution. In another vessel, PEO powder was mixed in $5 \mathrm{~g}$ of IPA and poured into water without any kind of stirring. Both vessels were stored at $5^{\circ} \mathrm{C}$ during 72 hours to allow sufficient dissolution. Then, the solutions were mixed and gently stirred. After that, the fluid remained at room temperature, $22^{\circ} \mathrm{C}$, during several hours before the experimental run. 
Without pre-shear, the critical speed for flow instability is random. The pre-shear consists in applying a constant shear rate slightly larger than the critical shear for instabilities for sufficiently long time until the torque reaches a constant value. It is assumed the pre-shear brings the solution to a steady state that allows reproducible results for the transition experiments. The pre-sheared solutions were only used once.

The rotation speed of the inner cylinder was varied linearly with a constant acceleration and deceleration $\Delta \Omega / \Delta t= \pm 5.2 \times 10^{-3} \mathrm{rad} / \mathrm{s}^{2}$. This ramping rate was selected so the dimensionless acceleration agrees to the criterion of Dutcher and Muller [12]: $\Delta R e / \Delta \tau \lesssim 0.60$ for the solvent. Moreover, as the polymer concentration increases, the viscosity of the solution also increases. Hence the viscous diffusion time decreases and viscous effects act faster. Consequently, the experiments conducted here can be considered in quasi-static conditions with respect to the viscous effects. Newtonian fluids in quasi-static acceleration/deceleration conditions are know to have zero hysteresis [12].

\subsection{Degradation}

Early studies [34] have shown that PEO solutions are susceptible to degradation due to mechanical, thermal, oxidative or ageing processes. Recently, Dutcher and Muller [14] showed that the relaxation time drops significantly over ten days for aqueous solutions of PEG and PEO whereas it remains almost constant for aqueous solutions of PEO in $47 \%$ glycerol. The present study circumvents this drawback by always making use of the fluids the third day after preparation. In order to test the maximum shear rate before breakdown of the polymer properties, a solution was sheared up to the highest shear rate reachable in our Taylor-Couette system $(\approx 6201 / \mathrm{s})$ in an acceleration-deceleration cycle while monitoring the torque. Figure 2 reports the torque as a function of shear rate for the $1000 \mathrm{ppm}$ solution and for the solvent. First, the transition to inertio-elastic turbulence is identified by an abrupt change in slope at low shear rate $(\approx 501 / \mathrm{s})$ and an increase in the amplitude of torque fluctuations. At $\dot{\gamma} \simeq 4001 / \mathrm{s}$ the level of torque fluctuations decreases significantly and the vortices resemble turbulent Taylor vortices as in a Newtonian solution. The following steps of the curve correspond to a change in the number of vortices that reduces the torque further [32]. During the deceleration, the torque significantly reduces down to the level of the solution without PEO. This, together with the fact that the torque fluctuations are reduced, suggests that the fluid has lost some of its viscoelastic 


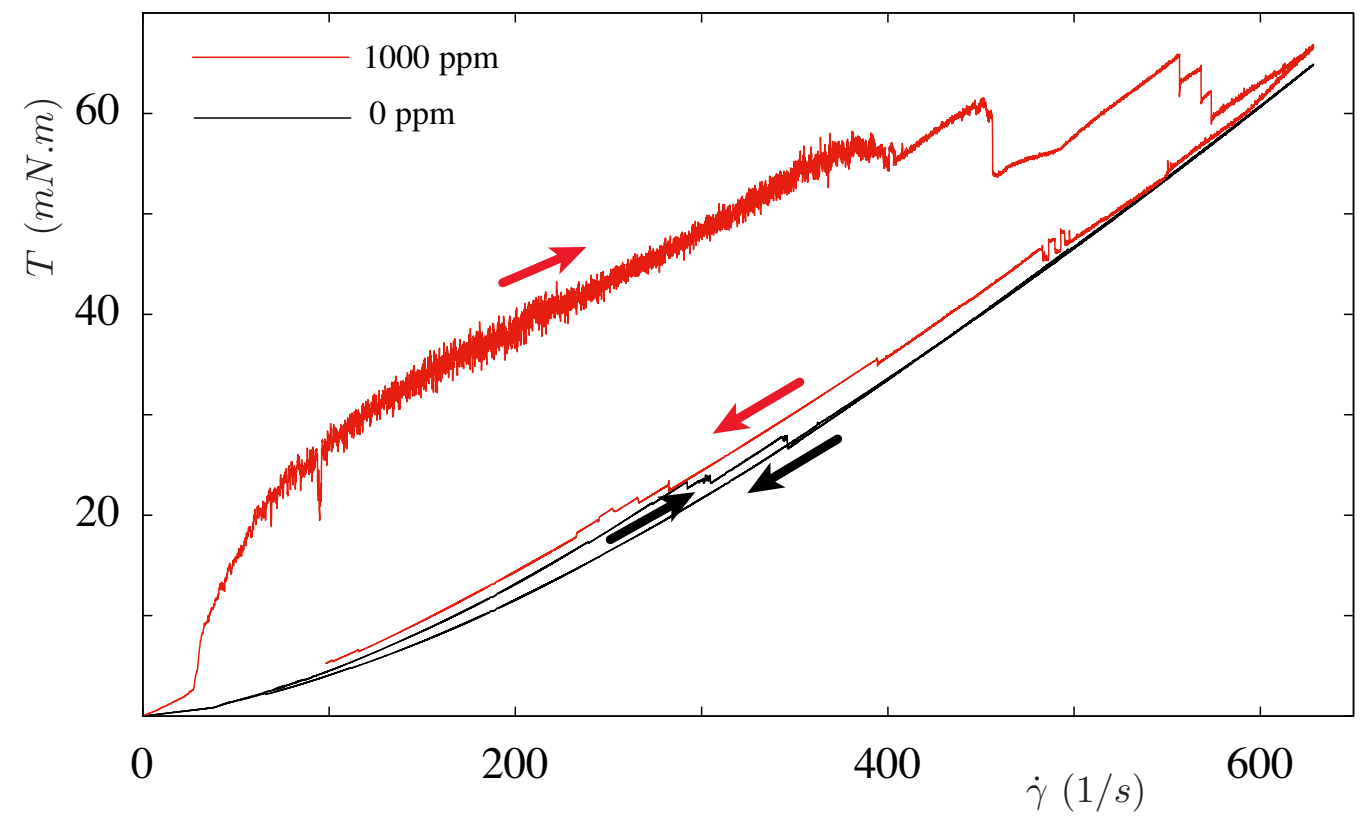

Figure 2: Torque as a function of shear rate for 0 and $1000 \mathrm{ppm}$ of PEO dissolved in $7 \%$ PEG aqueous solution during an acceleration and deceleration cycle in the Taylor-Couette geometry. These experiments were done without pre-shear. The up- and down-arrows indicate the acceleration and deceleration, respectively. 
properties. The experiments reported in the following were limited to shear rate below $85 \mathrm{~s}^{-1}$. Therefore, it is assumed that the degradation is negligible for $\dot{\gamma} \leq 85 \mathrm{~s}^{-1}$.

\section{Rheology}

\subsection{Shear rheology}

The polymer solutions were characterised using rotational and extensional rheometry. The values of the shear viscosity, $\mu$, as a function of the shear rate, $\dot{\gamma}$, are presented in figure 3 . As the concentration of PEO increases, the shear viscosity increases, but remains constant with respect to the shear rate. Only in the case of the solution with 1000 ppm of PEO, the shear thinning is significant and the shear viscosity can be fitted using a Carreau model:

$$
\mu=\mu_{\infty}+\left(\mu_{0}-\mu_{\infty}\right)\left[1+\left(\lambda_{C} \dot{\gamma}\right)^{2}\right]^{(n-1) / 2}
$$

where $\mu_{0}$ is the viscosity when $\dot{\gamma}$ tends to zero, $\mu_{\infty}$ is the viscosity when $\dot{\gamma}$ tends to infinity, $\lambda_{C}$ is the Carreau relaxation time and $n$ is the shearthinning index. Here $\mu_{0}=33.5 \mathrm{mPa} . \mathrm{s}, \mu_{\infty}=18.1 \mathrm{mPa} . \mathrm{s}, n=0.7$ and $\lambda_{C}=1.62 \mathrm{~s}$. The curve $\mu(\dot{\gamma})$ that fits the points is represented in figure 3 as a (red) continuous line, and is used to assess the viscosity in $R e$ and $N_{\omega}$ for 1000 ppm.

\subsection{Extensional rheology}

The extensional relaxation time, $\lambda_{e}$, was measured using a capillary breakup extensional rheometer $(\mathrm{CaBER})$ and recording the capillary thinning of a fluid filament joining two circular plates $[46,21]$. In figure 4(a), the evolution of the fluid filament diameter, $D(t)$, is monitored using a laser micrometer at the mid-plane between the end plates, when the upper one is rapidly displaced upwards. For Newtonian fluids, the diameter of the sample decreases linearly and no filament is observed. For the viscoelastic samples at slightly later times, the diameter decreases slower with increasing concentrations of PEO. To extract the extensional relaxation time, $\lambda_{e}$, the upper convected Maxwell constitutive model is used [46, 40, 21]:

$$
\frac{D(t)}{D_{0}} \propto \exp \left(-\frac{t}{3 \lambda_{e}}\right) \text {. }
$$




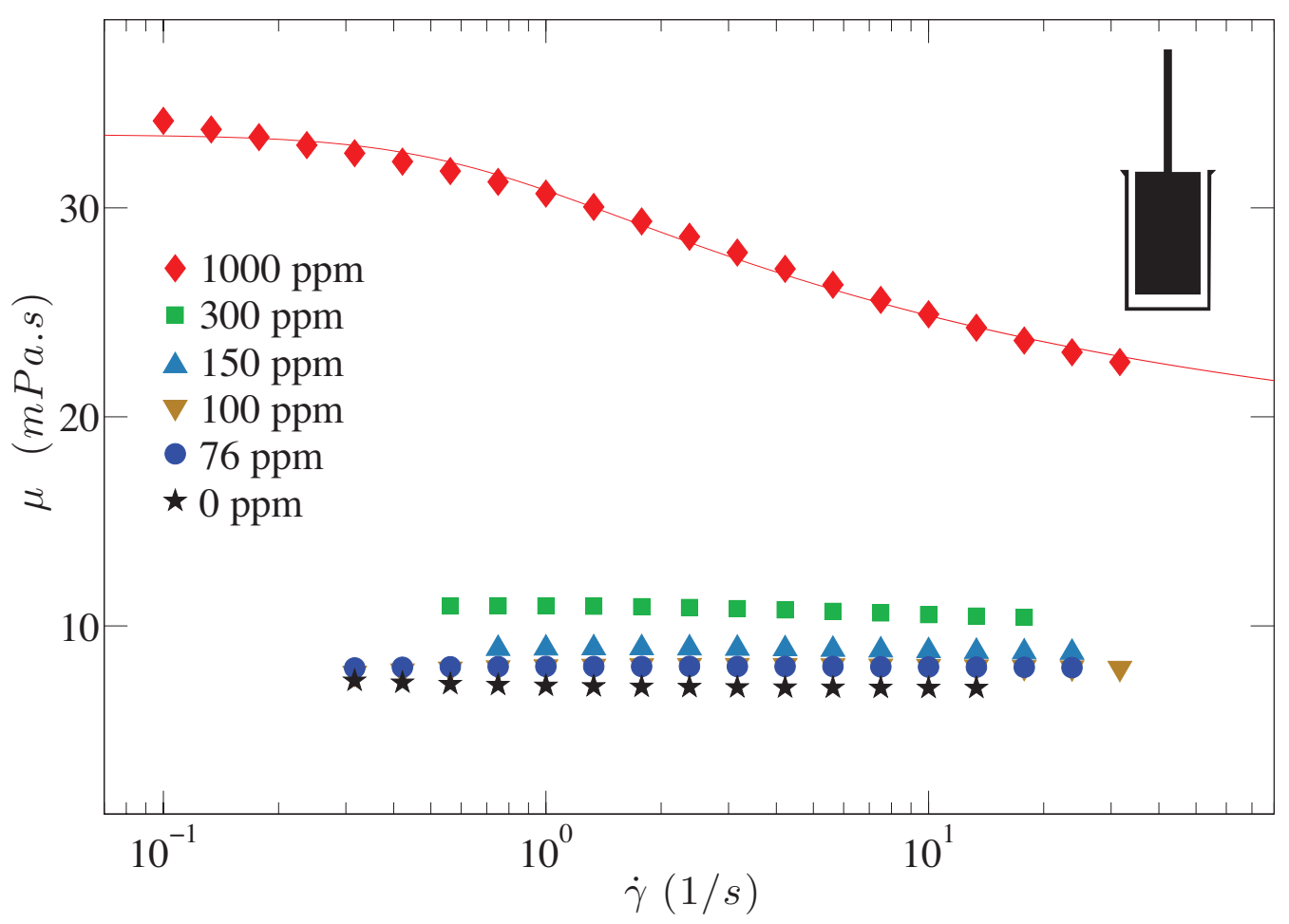

Figure 3: Shear viscosity, $\mu$, as a function of the shear rate, $\dot{\gamma}$, for different concentrations of PEO in 7\% PEG aqueous solution in the bob-cup geometry. The red continuous line corresponds to a fit by the Carreau model. 

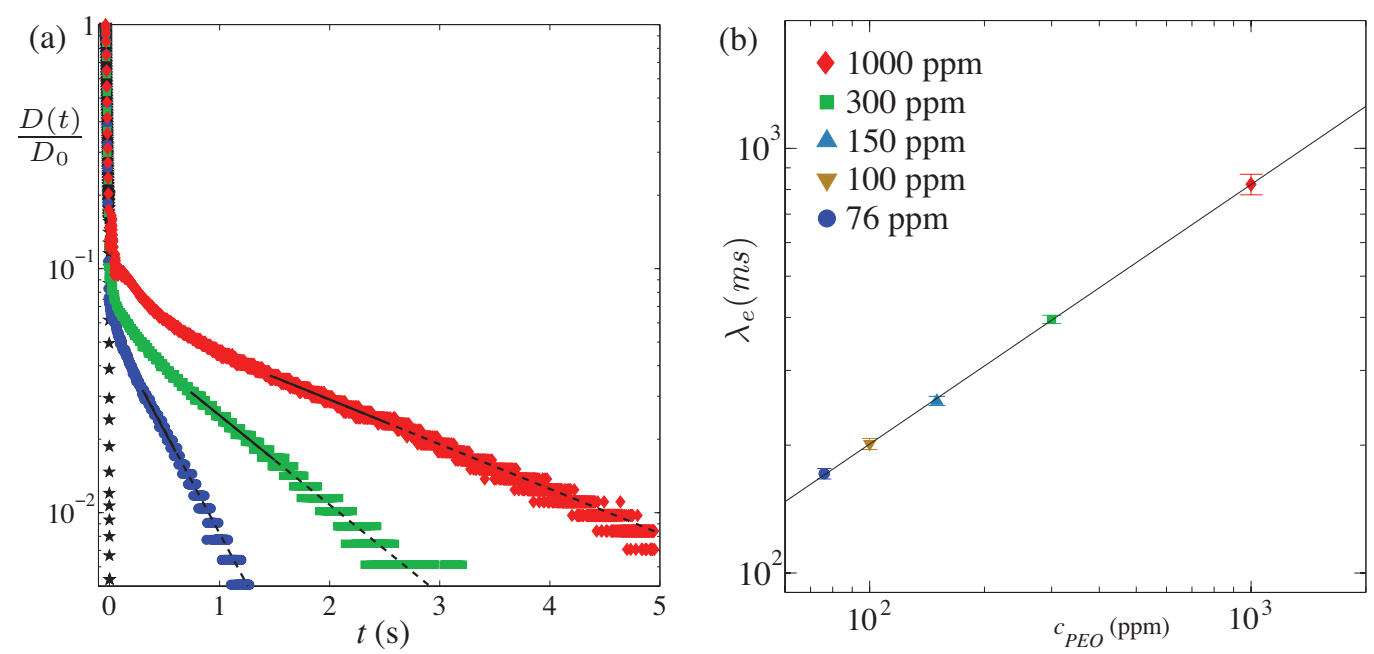

Figure 4: (a) Time evolution of the dimensionless diameter, $D(t) / D_{0}$, for different concentrations of PEO in 7\% PEG aqueous solution. The black stars correspond to the solvent. The continuous lines are fits of the thinning region in the elasto-capillary regime using (3) and dashed lines extrapolations of these fits. (b) Variation of relaxation time, $\lambda_{e}$, with the concentration of PEO. The error bars represent the standard deviation over three measurements and are smaller than the symbols. The black straight line is a power law fit with an exponent of 0.61 .

The figure 4(b) shows the extensional relaxation time versus POE concentration. In theory [40], the relaxation time should remain constant in dilute regime, yet $\lambda_{e}$ is found to increase monotonously with the concentration. The points have been fitted by an power-law function as $\lambda_{e} \propto c_{P E O}^{\beta}$, where the exponent $\beta=0.61$, which is close to $0.71 \pm 0.03$ found by Stelter et al. [46] for aqueous solutions of PEO of molar mass $4 \times 10^{6} \mathrm{~g} / \mathrm{mol}$.

Although the Taylor-Couette flow is shear dominated, the relaxation time used here is $\lambda_{e}$ as it is available for the whole range of concentrations. Note that $\lambda_{e}$ is shorter than the relaxation times from shear oscillatory tests $[2,6]$ and the Carreau parameter $\lambda_{C}$. However, $\lambda_{e}$ is longer than the relaxation time from normal force analysis [10].

The flow of polymer solutions is characterised by a stress tensor that can be expanded into two parts: the stress tensor of the solvent and the polymeric tensor. It follows that the total viscosity, $\mu$, can be interpreted as the sum of the solvent viscosity, $\mu_{s}$, and the polymeric viscosity, $\mu_{p}$, i.e., $\mu=\mu_{s}+\mu_{p}$. The elasticity of the fluid only has effects in the polymeric component of 
Table 1: Properties of the different PEO solutions dissolved in an aqueous solvent containing 7\% PEG whose shear viscosity, $\mu_{s}$, is $7.4 \mathrm{mPa}$.s. For the solution with $1000 \mathrm{ppm}$, the minimum and maximum values of $S$ and $E l$ correspond to the shear rate dependence of the viscosity.

\begin{tabular}{ccccccccccc}
\hline \hline $\begin{array}{c}c_{P E O} \\
(\mathrm{ppm})\end{array}$ & $\begin{array}{c}\mu \\
(\mathrm{mPa} . \mathrm{s})\end{array}$ & $S$ & $\begin{array}{c}\lambda_{e} \\
(\mathrm{~ms})\end{array}$ & $\begin{array}{c}\lambda_{C} \\
(\mathrm{~ms})\end{array}$ & $E l$ & $\begin{array}{c}\dot{\gamma}_{c} \\
(1 / \mathrm{s})\end{array}$ & $R e_{c}$ & $W i_{c}$ & $\mathcal{A}_{N_{\omega}(R e)}$ & $\mathcal{A}_{N_{\omega}(W i)}$ \\
\hline 76 & 8.07 & 0.09 & 171 & - & 0.06 & 42.5 & 133 & 7.5 & 1.0 & 0.05 \\
100 & 8.12 & 0.1 & 201 & - & 0.07 & 42 & 130 & 8.5 & - & - \\
150 & 8.95 & 0.21 & 254 & - & 0.09 & 45 & 127 & 11.5 & - & - \\
300 & 10.97 & 0.48 & 395 & - & 0.17 & 49 & 111 & 19 & 12.4 & 2.12 \\
1000 & $33.5-20.06$ & $1.71-3.53$ & 823 & 1620 & $0.71-1.09$ & 63 & 73 & 53 & 5.3 & 3.62 \\
\hline \hline
\end{tabular}

the stress, whereas the inertia has effects on both components. To quantify the importance of the polymeric stress to the total stress, the viscosity ratio is defined as $S=\mu_{p} / \mu_{s}=\left(\mu-\mu_{s}\right) / \mu_{s}$. Table 1 reports the values for the total shear viscosity, the viscosity ratio, the extensional relaxation time and the Elasticity number. The parameters $S$ and $E l$ can be used to determine the boundary between the inertia-dominated and the elasticity-dominated regimes.

\section{Results and discussion}

The results are presented in four parts. The first one is dedicated to the visualisations. The second one deals with torque measurements. The third one discusses the hysteresis phenomena. Finally, the fourth part is devoted to the diwhirls.

\subsection{Visualization}

Figure 5(a) presents the spatio-temporal diagram for a solution of 76 ppm of PEO with the inner cylinder accelerating. For low rotation speeds, the circular (Couette) flow and the Ekman vortices at the end gap of the cylinders develop. Then, as Re increases, these vortices evolve into a welldefined steady state of axisymmetric steady toroidal (Taylor) vortices that rapidly join at the centre of the cylinder. The critical $R e$ is at $133\left(\dot{\gamma}_{c}=42.5\right.$ $\mathrm{s}^{-1}$ ), indicating that the fluid is destabilised compared to the Newtonian case 

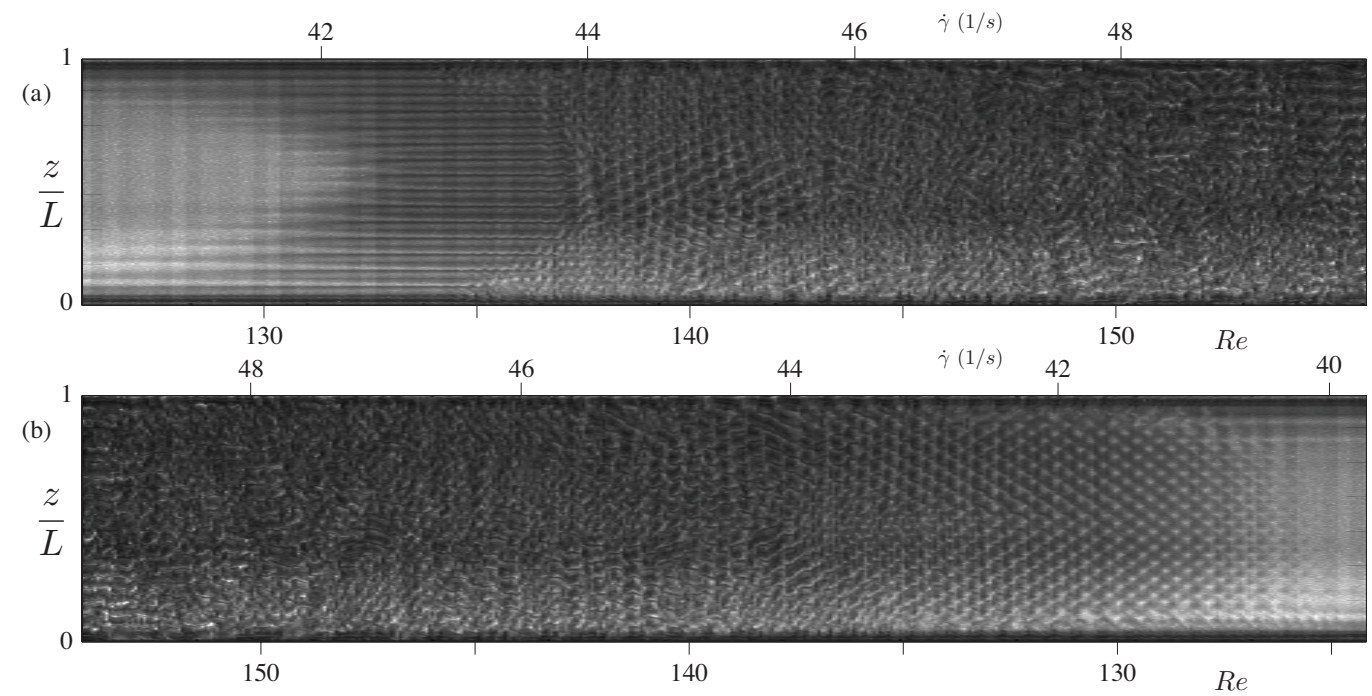

Figure 5: Spatio-temporal diagrams at the mid-plane of the gap over the whole height of the flow for $76 \mathrm{ppm}$ of PEO dissolved in 7\% PEG aqueous solution. (a) and (b) spatiotemporal diagrams of the flows solution during acceleration and deceleration, respectively.

for which the transition happens at $R e=135$. For the cases of 100 and 150 ppm the transitions occur at even lower $R e$, see $R e_{c}$ in table 1 . According to the non-monotonous behaviour of the stability boundaries found earlier [9], this $76 \mathrm{ppm}$ solution is not far from the intertio-elastic regime, as it destabilises the flow. Between $134<R e<137\left(43<\dot{\gamma}<44 \mathrm{~s}^{-1}\right)$ the Taylor vortices present weak oscillations that appear at the ends of the cylinder and propagate to the center, giving rise to disordered oscillating flow or noisy nonaxisymmetric standing waves, also called ribons, between $137<R e<143$ $(44<\dot{\gamma}<45.51 / \mathrm{s})[16,17,4,9]$. Beyond $\operatorname{Re}=143(\dot{\gamma}=45.51 / \mathrm{s})$ the space-time diagram shows a random pattern in which irregular lines emerge and disappear in short ranges of $R e$. This pattern is present for all the concentrations of PEO at high shear rates and is a characteristic of elastic instabilities [37]. A snapshot of this flow is shown in figure 8(b) as a pattern characterised by chaotically oscillating thin and entwined vortices. This pattern has also been named as "elastic turbulence" [16] or "elastically influenced turbulence" [13].

The deceleration ramp for the $76 \mathrm{ppm}$ solution is depicted in figure 5(b). Here, the disordered oscillating flow is also observed for $142>\operatorname{Re}>136$ 

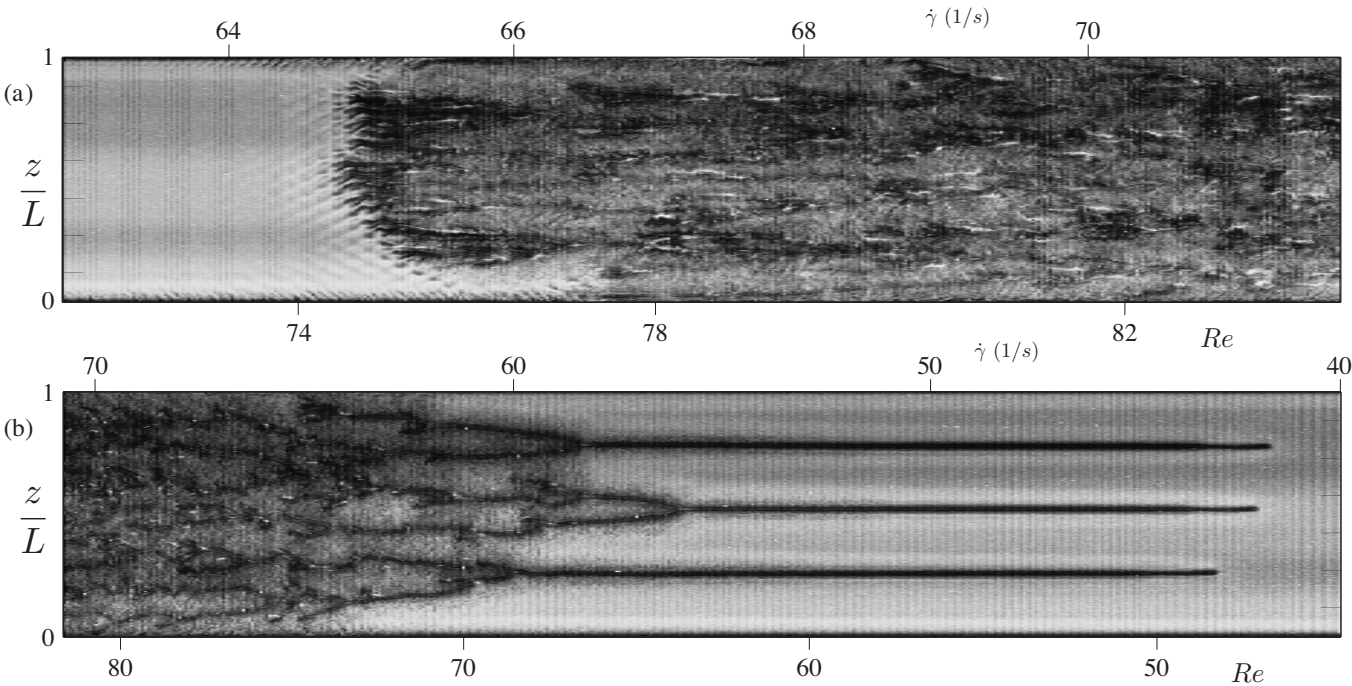

Figure 6: Spatio-temporal diagrams at the mid-plane of the gap over the whole height of the flow for $1000 \mathrm{ppm}$ of PEO dissolved in 7\% PEG aqueous solution. (a) and (b) spatio-temporal diagram of the flow during acceleration and deceleration, respectively. In (b) the black horizontal lines $(56>\dot{\gamma}>421 /$ s or $64>R e>48)$ correspond to diwhirls.

$\left(45.5>\dot{\gamma}>43.5 \mathrm{~s}^{-1}\right)$. However, it is not replaced by Taylor vortices but by symmetric standing waves $[16,17,4,9]$. For 100 and $150 \mathrm{ppm}$, Taylor vortices also also occur, but their range of existence reduces and the inertioelastic instabilities occur earlier. The corresponding diagrams are available in [33].

Figure 6(a) presents the spatio-temporal diagram of the flow for a solution of $1000 \mathrm{ppm}$ of $\mathrm{PEO}$ with the inner cylinder accelerating. A rapid transition from the laminar state to inertio-elastic turbulence is observed and no Taylor vortices are seen even close to the gap ends. Looking closely, short oblique standing waves are seen ahead of the inertio-elastic turbulence. These standing waves observed as diagonal lines in a narrow range around the transition are typical modes of high concentrations [9]. Figure 6(b) presents the spatio-temporal diagram of the flow with the inner cylinder decelerating. At $R e>81\left(\dot{\gamma}>70 \mathrm{~s}^{-1}\right)$ the inertio-elastic turbulent pattern is observed. Then, during deceleration, dark irregular and horizontal lines emerge giving rise to the flame pattern [4]. At $R e=64\left(\dot{\gamma}=56 \mathrm{~s}^{-1}\right)$ the branches of the flame pattern end up in three horizontal stationary lines, which correspond to three solitary pairs of vortices or diwhirls [17]. The average distance between 
the centres of the diwhirls is 7.5 times the gap. At $R e=47\left(\dot{\gamma}=42 \mathrm{~s}^{-1}\right)$, the diwhirls disappear from bottom to top. A snapshot of a three diwhirls pattern is shown in figure $8(\mathrm{e})$. The presence of diwhirls indicates that the $1000 \mathrm{ppm}$ fluid is clearly in the elastic regime as deduced by the analysis of $S(\simeq 1.71-3.53)$ and $E l(\simeq 0.71-1.09)$.

\subsection{Torque}

The torque data is shown in figure 7 as $N_{\omega}$ as a function of $R e$ and $W i$. In the laminar regime, $N_{\omega}$ is approximately constant and close to one. When $R e$ or $W i$ is small, $N_{\omega}$ is slightly shifted up due to the end effects that introduce an additional torque [32]. The end effects seem to be reduced for the $1000 \mathrm{ppm}$ solution, but it is not clear how the viscoelasticity affects them. At higher $R e$ or $W i$, there is a slight increase of the slope of $N_{\omega}$ before the abrupt increase of torque corresponding to the onset of inertio-elastic instabilities. This slight increase of torque was also observed in the study by Yi and Kim (1997) [50] and was attributed to the polymer contribution. As the shear rate exceeds a critical value, $\dot{\gamma}_{c}$, there is a rapid increase of the torque. Hence, as $E l$ increases, $R e_{c}$ decreases, as observed by previous authors for inertio-elastic flows [16,9]. This effect of $E l$ is also clear from figure 7(b) where the critical $W i$ increases with El. Table 1 summarises the critical values of $\dot{\gamma}_{c}, R e_{c}$ and $W i_{c}$. This torque or drag enhancement is due to the elastic properties of the fluid [50, 29]. It should be noted that $N_{\omega}$ fluctuates significantly for large value of $R e$ or $W i$ and $E l$ as observed by Groisman and Steinberg (2004) [19]. The amplitude of the fluctuations increases with the polymer concentration.

\subsection{Hysteresis}

The up- and down-arrows in figure 7 indicate the acceleration and deceleration protocol, respectively. The paths followed by $N_{\omega}$ present hysteresis loops as observed experimentally before by Groisman and Steinberg (2004) [19]. This hysteretic region is a signature of a subcritical transition between the laminar state $\left(N_{\omega} \simeq 1\right)$ and the regime where $N_{\omega}$ fluctuates. The flow with the smallest polymer concentration exerts almost the same torque transfer when accelerating and decelerating, although the flow patterns are different: horizontal (Taylor-like) vortices upwards (figure 5(a)) and intertwined oblique standing waves downwards (figure 5(b)).

The dimensionless areas enclosed between the acceleration and deceleration curves in figure $7(\mathrm{a})$ and (b) are defined as $\mathcal{A}_{N_{\omega}(R e)}$ and $\mathcal{A}_{N_{\omega}(W i)}$, re- 

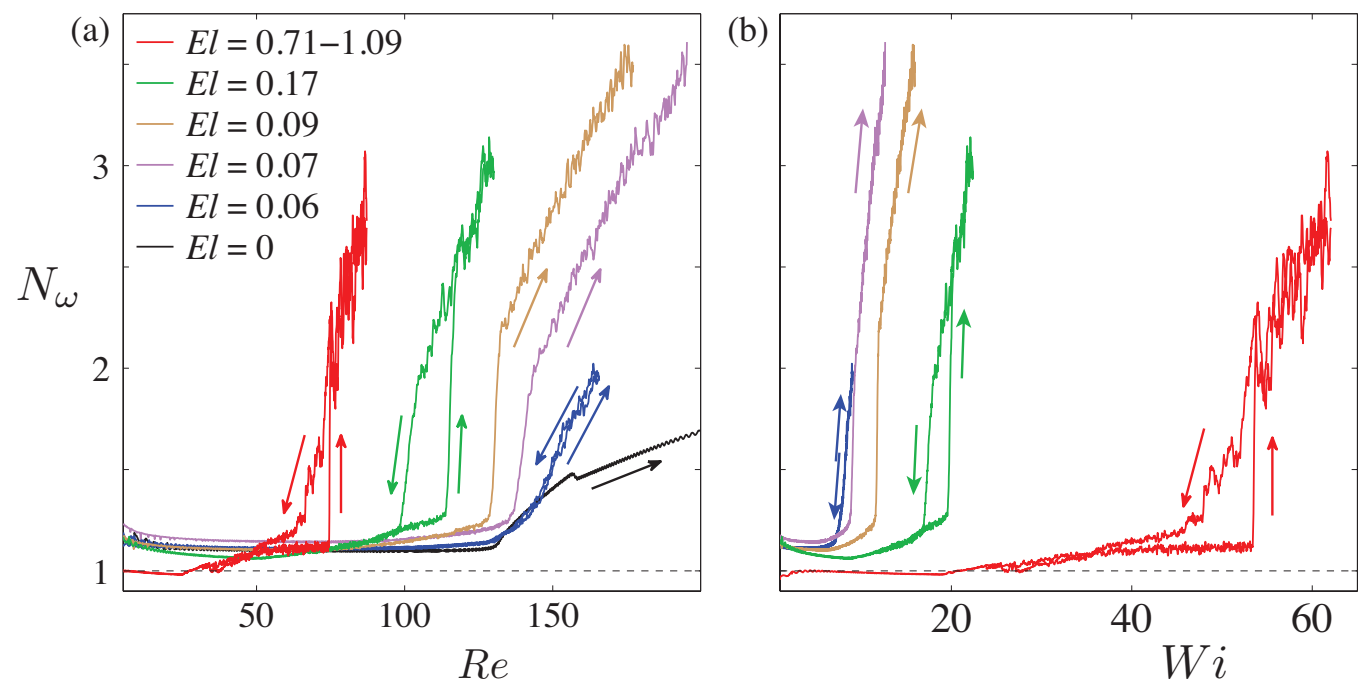

Figure 7: (a) $N_{\omega}$ as a function of the $R e$ and (b) $N_{\omega}$ as a function of the $W i$ for solutions with different $\mathrm{El}$ or concentrations of $\mathrm{PEO}$ dissolved in $7 \% \mathrm{PEG}$ aqueous solution. The up- and down-arrows indicate the acceleration and deceleration, respectively. Down-curves for $E l=0.07$ and 0.09 are omitted.

spectively. The values of $\mathcal{A}_{N_{\omega}(R e)}$ and $\mathcal{A}_{N_{\omega}(W i)}$ are given in table 1 for the 76 , 300 and $1000 \mathrm{ppm}$ (data for 100 and $150 \mathrm{ppm}$ are missing). These indicate that the amount of hysteresis seem to increase with the elasticity, although non-monotonously. Indeed, the area for low polymer concentration (76 ppm or $E l=0.06$ ) is presenting a low area value (small hysteresis) and for the highest concentration case $(1000 \mathrm{ppm}$ or $E l \geq 0.71), \mathcal{A}_{N_{\omega}(R e)}$ is not maximum. Such hysteresis loop has also been found in numerical simulations by Thomas et al. (2009) [47]. Both in the present experiments and in the simulations, the flame pattern emerges when decelerating or reducing $W i$ or $R e$ from 72 to 65 (see figure 6(b) for $62>\dot{\gamma}>56 \mathrm{~s}^{-1}$ ). The associated torque is shown in figure 7 (b) for $53>W i>45$ and appears as "staircases". In the experiments, an anticlockwise hysteresis loop is observed, where the upper branch corresponds to the flame pattern. However, in the simulations, the initial state is oscillatory strips, which remain stable when increasing $W i$, and have larger torque than the flame pattern. Hence the hysteresis loop predicted from the simulations is in the clockwise direction, contrary to the experiments. 
(a)

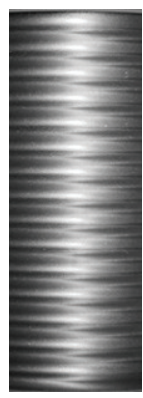

(b)

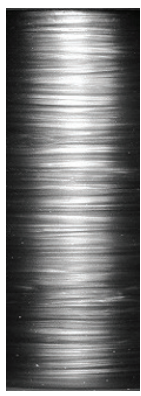

(c)

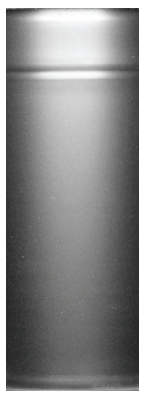

(d)

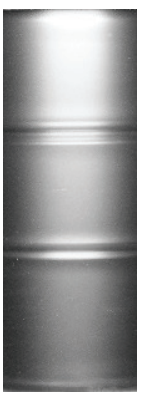

(e)

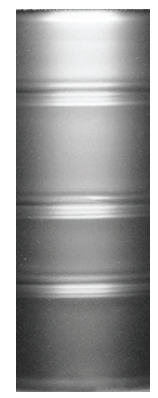

(f)

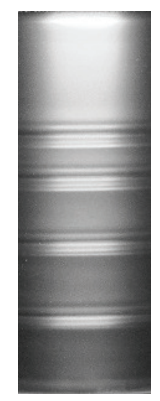

$(\mathrm{g})$

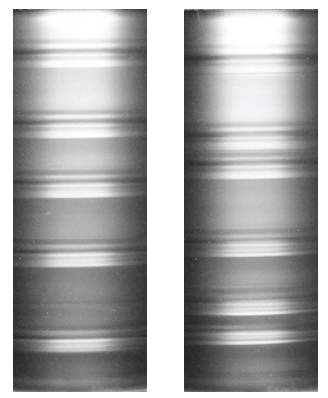

Figure 8: Snapshots over the whole height of the flow. (a) Newtonian fluid containing 30 Taylor vortices. The others are for mixtures containing $1000 \mathrm{ppm}$ of PEO dissolved in 7\% PEG aqueous solution and present: (b) inertio-elastically turbulent flow, (c-h) flows containing 1 to 6 diwhirls, from left to right, respectively.

\subsection{Diwhirls}

During deceleration, coming back from the inertio-elastic turbulent regime, the latest stages of the hysteretic region gives rise to steady vortices called diwhirls [17]. Here, these flow solutions was observed for the largest concentration, $1000 \mathrm{ppm}$, coming back from an inertio-elastic turbulent flow at $R e \approx 50$ or $W i \approx 62$. These steady vortices have been found in the form of 1 to 6 pairs and are depicted in figure 8(c-h). These diwhirls can be compared with the 30 Taylor vortices flow state and the inertio-elastic turbulent flow in figures 8(a) and (b), respectively. Clearly, they have longer wavelength than Taylor vortices. Note that the diwhirls are not equally distributed along the vertical axis, which suggest a random process for the formation mechanism of these vortices. In practice, the numbers of diwhirls is controlled by the deceleration rate of the inner cylinder as quantified in figure 9(a). Using the constant deceleration of $\Delta \Omega / \Delta t=0.1 \times \Delta \dot{\gamma} / \Delta t=-5.2 \times 10^{-3} \mathrm{rad} / \mathrm{s}^{2}$, three diwhirls are observed (see figure $8(\mathrm{~d})$ and figure $8(\mathrm{e})$ ). When using faster deceleration, the number of diwhirls is larger. An alternative procedure to obtain 1 or 2 diwhirls is to decrease the speed of the inner cylinder until the diwhirls start to disappear. Once the desired number of diwhirls is reached, the inner cylinder is accelerated to a speed that can sustain it. Furthermore, the existence of diwhirls leads to an additional torque with respect to the laminar torque. It has been measured at a constant speed, $R e=51$ or $W i=37$, for several minutes and is represented in figure 9 (b) as the additional torque 

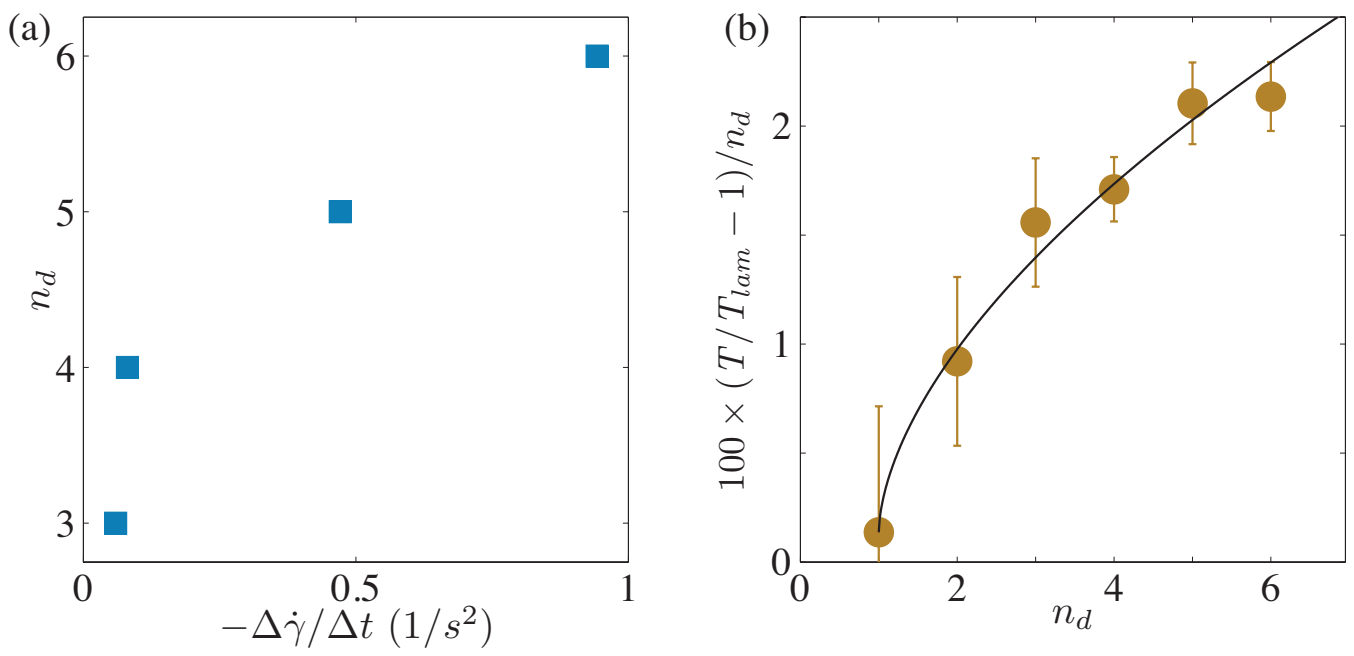

Figure 9: (a) Dependency of the numbers of diwhirls, $n_{d}$, on the deceleration of the inner cylinder, $-\Delta \dot{\gamma} / \Delta t$. (b) Additional dimensionless torque over the laminar per numbers of diwhirls, $n_{d}$, of flows containing six different numbers of diwhirls at $R e=51$ or $W i=37$. The black line represents a fit described in the text.

compared to the laminar, $\bar{T}-T_{\text {lam }}$, per number of diwhirls, $n_{d}$, divided by the laminar torque. Here, the laminar torque, $T_{\text {lam }}$, is the torque of the flow without any diwhirl. The increase of this relationship with $n_{d}$ indicates that the additional torque that each individual diwhirls needs to be sustained depends on the numbers of diwhirls. This could be also explained relating this increase of torque to the distance between diwhirls, so the closer they are the more torque they need. The variation of the torque with the number of diwhirls is given by the following fit: $\left(\bar{T} / T_{\text {lam }}-1\right) / n_{d}=A+B\left(n_{d}-1\right)^{\alpha}$, where $A$ represents the dimensionless torque over the laminar per number of diwhirls for a flow containing one diwhirl. The term $B$ is related to the influence of the numbers of diwhirls on the additional torque over the laminar. The exponent $\alpha$ indicates how strong is the dependency of the torque on the numbers of diwhirls. Here $\alpha \simeq 3 / 5, A=1.37 \times 10^{-3}$ and $B=8.4 \times 10^{-3}$. Although deceleration rate is smaller than any elastic relaxation time, the fluid selects the number of diwhirls accordingly. This suggests that a range of relaxation times controlling the behaviour of the fluid [43] and support the ideas of relaxation times dependent on shear rate as measured in previous studies [7]. 


\section{Conclusions}

The effect of long-chain polymer addition over a wide range of concentrations on the torque in Taylor-Couette flow has been investigated. The shear and the extensional properties of the solutions have been quantified when degradation is assumed negligible. Flow visualisations and torque measurements have been performed simultaneously during slow accelerationdeceleration cycles.

At low concentration (76 ppm), the transition involves a sequence of flow pattern: azimuthal flow, Taylor vortices, disordered oscillating flow and inertio-elastic turbulence in the accelerating phase. In the decelerating phase, inertio-elastic turbulence, disordered oscillating flow, standing waves and azimuthal flow are observed. For large concentration (1000 ppm), the transition involve azimuthal flow, standing waves and inertio-elastic turbulence in the accelerating phase. During the deceleration, inerto-elastic turbulence, flame pattern, diwhirls and azimuthal flow were observed. These transitions, that were reported before $[4,19,14]$, are again observed and the associated torque is given. The hysteresis of the flow patterns and the torque behaviour is quantified and may be related to subcritical flow instabilities [35] or conformational hysteresis [44], which corresponds to stretched polymer configurations. In table 1, the properties of the fluids, the critical values of the shear rate, the Reynolds number and Weissenberg number, and the hysteresis coefficient defined by the area of the hysteretic region of the torque behaviour are reported. This hysteresis seems to increase with the elasticity number. This effect could be used in dampers for passive control.

During the deceleration, different numbers of solitary vortex pairs or diwhirls are observed depending on the deceleration rate of the inner cylinder. The number of diwhirls can be tuned by the deceleration rate, yet they seem to form unarranged as their separation length is random. The torque they exert has been measured and is dependent on the number of diwhirls.

\section{Acknowledgments}

The work was partly financed through the French Agence Nationale de la Recherche, through the program Investissement d'Avenir (ANR-10-LABX09-01), LabEx EMC3, the CPER Haute-Normandie and the European Fund for Regional Development. The authors are grateful to Olivier Crumeyrolle 
and Innocent Mutabazi for discussions. JP thanks Morton Denn for pointing out several useful references.

[1] R.M. Andrade, A.S. Pereira, E.S. Soares, Drag increase at the very start of drag reducing flows in a rotating cylindrical double gap device, J. NonNewtonian Fluid Mech. 212 (2014) 73-79.

[2] O. Arnolds, H. Buggisch, D. Sachsenheimer, N. Willenbacher, Capillary breakup extensional rheometry (CaBER) on semi-dilute and concentrated polyethyleneoxide (PEO) solutions, Rheol. Acta 49 (2010) 1207-1217.

[3] F.J. Bailey, Poly(ethylene oxide), Academic Press, Inc., 1976.

[4] B.M. Baumert, S.J. Muller, Axisymmetric and non-axisymmetric elastic and inertio-elastic instabilities in Taylor-Couette flow, J. Non-Newtonian Fluid Mech. 1 (1999) 33-69.

[5] G.S. Beavers, D.D. Joseph, Tall Taylor cells in polyacrylamide solutions, Phys. Fluids 17 (1974) 650-651.

[6] F. Bossard, N. El Kissi, A. D'Aprea, F. Alloin, J.-Y. Sanchez, A. Dufresne, Influence of dispersion procedure on rheological properties of aqueous solutions of high molecular weight PEO, Rheol. Acta 49 (2010) $529-540$.

[7] L. Casanellas, M.A. Alves, R.J. Poole, S. Lerouge, A. Lindner, The stabilizing effect of shear thinning on the onset of purely elastic instabilities in serpentine microflows, Soft Matter 12 (2016) 6167.

[8] C. Clasen, J. Plog, W.-M. Kulicke, M. Owens, C. Macosko, L. Scriven, M. Verani, G.H. McKinley, How dilute are dilute solutions in extensional flows? J. Rheol. 50 (2006) 849-881.

[9] O. Crumeyrolle, I. Mutabazi, M. Grisel, Experimental study of inertioelastic Couette-Taylor instability modes in dilute and semidilute polymer solutions, Phys. Fluids 14 (2002) 1681-1688.

[10] O. Crumeyrolle, N. Latrache, A. Ezersky, I. Mutabazi, Instability modes observed in a viscoelastic Couette-Taylor flow, Mécanique \& Industries 4 (2003) 397-409. 
[11] M. Denn, J. Roisman, Rotational stability and measurement of normal stress functions in dilute polymer solutions, AIChE J. 15 (1969) 454-459.

[12] C.S. Dutcher, S.J. Muller, Spatio-temporal mode dynamics and higher order transitions in high aspect ratio Newtonian Taylor-Couette flows, J. Fluid. Mech. 641 (2009) 85-113.

[13] C.S. Dutcher, S.J. Muller, Effects of weak elasticity on the stability of high Reynolds number co-and counter-rotating Taylor-Couette flows, J. Rheol. 55 (2011) 1271--1295.

[14] C.S. Dutcher, S.J. Muller, Effects of moderate elasticity on the stability of co-and counter-rotating Taylor-Couette flows, J. Rheol. 57 (2013) 791-812 .

[15] W.W. Graessley, Polymer chain dimensions and the dependence of viscoelastic properties on concentration, molecular weight and solvent power, Polymer 21 (1980) 258--262.

[16] A. Groisman, V. Steinberg, Couette-Taylor flow in a dilute polymer solution, Phys. Rev. Lett. 77 (1996) 1480.

[17] A. Groisman, V. Steinberg, Solitary vortex pairs in viscoelastic Couette flow, Phys. Rev. Lett. 78 (1997) 1460.

[18] A. Groisman, V. Steinberg, Elastic vs. inertial instability in a polymer solution flow, EPL 43 (1998) 165.

[19] A. Groisman, V. Steinberg, Elastic turbulence in curvilinear flows of polymer solutions, New J. Phys. 6 (2004) 29.

[20] R. Haas, K.Bühler, Einfluß nichtnewtonscher stoffeigenschaften auf die Taylorwirbelströmung, Rheol. Acta 28 (1989) 402-413.

[21] S.J. Haward, V. Sharma, C.P. Butts, G.H. McKinley, S.S. Rahatekar, Shear and extensional rheology of cellulose/ionic liquid solutions, Biomacromolecules 13 (20112) 1688-1699.

[22] F. Kelai, Etude expérimentale des intabilités viscoélastiques dans le système de Couette-Taylor, Doctoral Thesis, Le Havre University, France, 2011. 
[23] W.-M. Kulicke, C. Clasen, Viscosimetry of polymers and polyelectrolytes, Springer, 2004.

[24] K. Kumar, M.D. Graham, Finite-amplitude solitary states in viscoelastic shear flow: computation and mechanism, J. Fluid Mech. 443 (2001) 301328.

[25] M. Lange, B. Eckhardt, Vortex pairs in viscoelastic Couette-Taylor flow, Phys. Rev. E 64 (2001) 027301.

[26] R.G. Larson, E.S.G. Shaqfeh, S.J. Muller, A purely elastic instability in Taylor-Couette flow, J. Fluid Mech. 218 (1990) 573-600.

[27] N. Latrache, O. Crumeyrolle, I. Mutabazi, Transition to turbulence in a flow of a shear-thinning viscoelastic solution in a Taylor-Couette cell, Phys. Rev. E 86 (2012) 056305.

[28] N. Latrache, N. Abcha, O. Crumeyrolle, I. Mutabazi, Defect-mediated turbulence in ribbons of viscoelastic Taylor-Couette, Phys. Rev. E 93 (2016) 056305.

[29] N. Liu, B. Khomami, Elastically induced turbulence in Taylor-Couette flow: direct numerical simulation and mechanistic insight, J. Fluid Mech. 737 (2013) R4.

[30] N. Liu, B. Khomami, Polymer-induced drag enhancement in turbulent Taylor-Couette flows: Direct numerical simulations and mechanistic insight, Phys. Rev. Lett. 111 (2013) 114501.

[31] S. Majumdar, A.K. Sood, Universality and scaling behavior of injected power in elastic turbulence in wormlike micellar gel, Phys. Rev. E 84 (2011) 015302.

[32] B. Martínez-Arias, J. Peixinho, O. Crumeyrolle, I. Mutabazi, Effect of the number of vortices on the torque scaling in Taylor-Couette flow, J. Fluid Mech. 748 (2014) 756-767.

[33] B. Martínez-Arias, Torque measurement in turbulent Taylor-Couette flow, Thèse de doctorat, Université Le Havre Normandie, France, 2015.

[34] C.W. McGary, Degradation of poly(ethylene oxide), J. Polym. Sci. 46 (1960) 51--57. 
[35] H. Mohammadigoushki, S.J. Muller, Inertio-elastic instability in TaylorCouette flow of a model wormlike micellar system, J. Rheol. 61(4) (2017) 683-696

[36] A.N. Morozov, W. van Saarloos, Subcritical finite-amplitude solutions for plane Couette flow of viscoelastic fluids, Phys. Rev. Lett. 95 (2005) 024501.

[37] S. Muller, R. Larson, E. Shaqfeh, A purely elastic transition in TaylorCouette flow, Rheol. Acta 28 (1989) 499-503.

[38] A.S. Pereira, E.S. Soares, Polymer degradation of dilute solutions in turbulent drag reducing flows in a cylindrical double gap rheometer device, J. Non-Newtonian Fluid Mech. 179-180 (2012) 9-22.

[39] A.M. Philippe, C. Baravian, M. Jenny, F. Meneau, L.J. Michot, TaylorCouette instability in anisotropic clay suspension measured using smallangle X-ray scattering, Phys. Rev. Lett. 108 (2012) 254501.

[40] L.E. Rodd, T.P. Scott, J.J. Cooper-White, G.H. McKinley, Capillary break-up rheometry of low-viscosity elastic fluids, Appl. Rheol 15 (2005) $12-27$.

[41] L.E. Rodd, J. Cooper-White, D. Boger, G. McKinley, Role of the elasticity number in the entry flow of dilute polymer solutions in microfabricated contraction geometries, J. Non-Newtonian Fluid Mech. 143 (2007) 170-191.

[42] M. Rubinstein, R.H. Colby, Polymer physics, Oxford University Press, 2003.

[43] D.G. Satchwell, T. Mullin, Bifurcation phenomena in Taylor-Couette flow of elastic polymer solutions, Proc. R. Soc. Lond. A 457 (2001) 25672583.

[44] C.M. Schroeder, H.P. Babcock, E.S.G. Shaqfeh, S. Chu, Observation of polymer conformation hysteresis in extensional flow, Science 301 (2003) 1515-1519.

[45] H. Singh, C.A.T. Suazo, A. Liné, Log law of the wall revisited in TaylorCouette flows at intermediate Reynolds numbers, Phys. Rev. E 94 (2016) 053120 . 
[46] M. Stelter, G. Brenn, A. Yarin, R. Singh, F. Durst, Validation and application of a novel elongational device for polymer solutions, J. Rheol. 44 (2000) 595-616.

[47] D. Thomas, B. Khomami, R. Sureshkumar, Nonlinear dynamics of viscoelastic Taylor-Couette flow: effect of elasticity on pattern selection, molecular conformation and drag, J. Fluid Mech. 620 (2009) 353-382.

[48] K. Watanabe, S. Sumio, S. Ogata, Formation of Taylor vortex flow of polymer solutions, J. Fluids Eng. 128 (2006) 95-100.

[49] F. Wendt, Turbulente strömungen zwischen zwei rotierenden konaxialen zylindern, Ing.-Arch. 4 (1933) 577-595.

[50] M.-K. Yi, C. Kim, Experimental studies on the taylor instability of dilute polymer solutions, J. Non-Newtonian Fluid Mech. 72 (1997) 113-139. 